\title{
Comportamiento hidrológico de una pequeña cuenca de uso silvopastoril en el sureste de Coahuila
}

Miguel A. Velásquez Valle ${ }^{1}$

Gerardo Esquivel Arriaga²

Ignacio Sánchez Cohen ${ }^{2}$

David Castillo Quiroz ${ }^{1 *}$

Antonio Cano Pineda ${ }^{1}$

Ramón Gutiérrez Luna ${ }^{3}$

${ }^{1}$ Campo Experimental Saltillo, CIR Noreste. INIFAP. México.

${ }^{2}$ Centro Nacional de Investigación Disciplinaria en Relación Agua-Suelo-PlantaAtmósfera. INIFAP. México.

${ }^{3}$ Campo Experimental Zacatecas. CIR Norte-Centro. INIFAP. México.

*Autor por correspondencia, correo-e: castillo.david@inifap.gob.mx 


\section{Resumen:}

El deterioro de los ecosistemas forestales pone en riesgo el almacenamiento y flujo del agua de lluvia. En el estado de Coahuila, existen 450 mil hectáreas de superficie forestal, las cuales son un área de captación de agua de lluvia. Para lograr un mayor aprovechamiento de este recurso es indispensable el monitoreo continuo de variables hidrológicas en las cuencas, principalmente, donde existe incertidumbre climática. El objetivo del presente estudio fue documentar el comportamiento hidrológico de una pequeña cuenca de uso ganadero-forestal, a partir del monitoreo de variables y el empleo de un modelo hidrológico de parámetros semidistribuidos espacialmente de la Cuenca Experimental del INIFAP, ubicada en el municipio Saltillo, Coah. Con registros históricos, cuantificados durante 2016 de precipitación pluvial se realizó el análisis de la respuesta hidrológica. Se utilizó el modelo WIN TR-55 para explicar la ausencia de escurrimiento, en función de parámetros semidistribuidos. Con los datos pluviométricos de 2016, se observó que las áreas de aportación de flujo superficial son las laderas y que la parte baja de la misma (el valle) actúa como un área buffer que restringe el escurrimiento superficial hacia la estación de aforo. En función a las simulaciones con el Modelo WIN TR - 55, se requiere una lámina precipitada diaria (máxima en 24 horas) mayor a $65 \mathrm{~mm}$ para registrar flujo superficial en la estación de aforo.

Palabras clave: Área fuente, curva numérica, escurrimiento superficial, modelo WIN TR - 55, monitoreo de precipitación, procesos hidrológicos.

Fecha de recepción/Reception date: 5 de marzo de 2017

Fecha de aceptación/Acceptance date: 26 de agosto de 2017. 
La superficie forestal de México es aproximadamente de 138 millones de hectáreas, lo que representa $70.4 \%$ del territorio nacional. De esta, $24.2 \%$ corresponde a los bosques templados (DOF, 2014). Entre otros beneficios, el dosel forestal filtra el agua de lluvia y regula el flujo del agua en el ecosistema. Sin embargo, los recursos forestales de manera continua están expuestos a agentes que causan su deterioro, como ecosistema. Entre los principales agentes que lo provocan se citan a los incendios (Rodríguez-Trejo y Fulé, 2003), las plagas y enfermedades (Sánchez et al., 2003), los cambios de uso de suelo (Torres-Rojo y Flores-Xolocotzi, 2014) y, sobretodo, la tala inmoderada.

El deterioro de los ecosistemas forestales conlleva, simultáneamente, a la alteración de procesos físicos como la infiltración y generación del escurrimiento que determinan su funcionamiento y sostenibilidad. Por ejemplo, la ocurrencia de un incendio puede alterar los procesos de intercepción, infiltración, evapotranspiración, almacenamiento de la humedad en el suelo y el flujo del agua sobre la superficie del terreno (DeBano, 2009).

Impactos hidrológicos. La manipulación de la vegetación de las áreas forestales ha causado modificaciones hidrológicas. Algunas causas son la reducción del dosel de los bosques e incrementos en la evapotranspiración (Swindel et al., 1983; Cui et al., 2012). Factores ecológicos como la cantidad y estructura del dosel de los árboles reducen las pérdidas de agua y la producción de sedimentos (Zheng et al., 2008).

Por otro lado, se ha registrado que los escurrimientos superficiales en áreas forestales dependen, parcialmente, de la profundidad del suelo, su estructura y el grado de saturación antes de ocurrir la precipitación (Yu et al., 2015); así como del mantillo orgánico sobre el suelo y las propiedades del mismo (Zheng et al., 2008).

En cuencas forestales, otro aspecto hidrológico relevante, aunque no está considerado en este estudio, es la presencia de flujo preferencial, principalmente en aquellos sitios donde el valor de la conductividad hidráulica del suelo es alto, por la 
continuidad y densidad de los macroporos ocasionados por el tamaño de las raíces de especies arbóreas en la matriz del suelo (Alaoui et al., 2010).

Existen métodos específicos para determinar los cambios en las tendencias de las variables hidrológicas debidos a la manipulación de la vegetación; por ejemplo, el método de hidrograma unitario (Černohous y Kovář, 2009). Sin embargo, la herramienta más utilizada para simular y predecir el comportamiento hidrológico en cuencas son los modelos matemáticos (Gholami, 2013; Hlásny et al., 2015). Chen et al. (2013) evaluaron diferentes tipos de modelos (empíricos y físicamente basados) para determinar el impacto de incendios en una cuenca forestal en procesos como evapotranspiración, flujo superficial, flujo en canales y flujo en áreas sub-superficiales saturadas y no saturadas.

Uno de los modelos determinísticos utilizados para cuantificar el impacto hidrológico en cuencas con diferente uso de suelo, es el WIN TR - 55 (USDA-NRCS, 1986), el cual es empírico y con parámetros semidistribuidos para cuencas no mayores a 6500 ha. Para el cálculo de la lámina escurrida se utiliza el Método de Curva Numérica propuesto por el SCS (USDA-SCS, 1972); además asume una condición de humedad antecedente II (promedio) y asigna parámetros por subcuenca de superficie, curva numérica (tipo de suelo, condición hidrológica y uso de suelo) y de hidráulica de los canales (pendiente, coeficiente de Manning, ancho de la base del canal, relación pendiente-talud y tipo de cobertura), por evento de precipitación.

Importancia del monitoreo hidrológico. De la superficie del estado de Coahuila (15 067115 ha) se cita que tan solo $4.1 \%$ de su vegetación corresponde a bosques de coníferas, de coníferas - latifoliadas y de latifoliadas, según el Inventario Estatal Forestal y de Suelos (Semarnat-Conafor, 2013). Dicha superficie es un área de captación y almacenamiento de agua de lluvia. El líquido captado y almacenado constituye una fuente de abastecimiento para uso residencial, recreativo e industrial. Desde el punto de vista del balance hidrológico en cuencas de los bosques, en la entidad no se cuenta con referencias recientes; sin embargo, 
cabe resaltar que el tema es considerado como uno de los objetivos del Programa Especial del Agua 2011-2017 (Gobierno de Coahuila, 2012), en el cual se indica que es importante "Asegurar el conocimiento del ciclo hidrológico en la entidad y propiciar la investigación científica para el uso sustentable del agua".

Así, para lograr un mayor aprovechamiento de este recurso es indispensable el monitoreo continuo de variables hidrológicas, con el propósito de tener fundamentos técnicos de procesos físicos como la generación del flujo superficial para la toma de decisiones en el manejo de los recursos naturales (Sánchez, 2005; Cui et al., 2012).

El objetivo del presente estudio fue documentar el comportamiento hidrológico de una pequeña cuenca ganadera-forestal, mediante el monitoreo de variables y el uso de un modelo matemático de parámetros semidistribuidos, lo anterior para explicar el comportamiento hidrológico de la Cuenca Experimental Saltillo (CES) del INIFAP ubicada en la región sur Saltillo, Coah durante 2016.

\section{Área de estudio}

La Cuenca Experimental Saltillo (CES) se localiza en la región hidrológica BravoConchos (RH24Be), en el municipio de Saltillo, entre los meridianos 101 $00^{\prime} 30^{\prime \prime}$ longitud oeste y los paralelos $25^{\circ} 09^{\prime} 00^{\prime \prime}$ latitud N, y tiene una superficie de 47.2 hectáreas (Figura 1). La CES se ubica en la región conocida como Sierra de Zapalinamé, con una superficie de 45226.8 hectáreas (Gobierno del Estado de Coahuila, 1998). Esta microregión cuenta con una extensa y variada vegetación, entre la que destacan el bosque de pino piñonero, de encinos y matorrales xerófilos. 


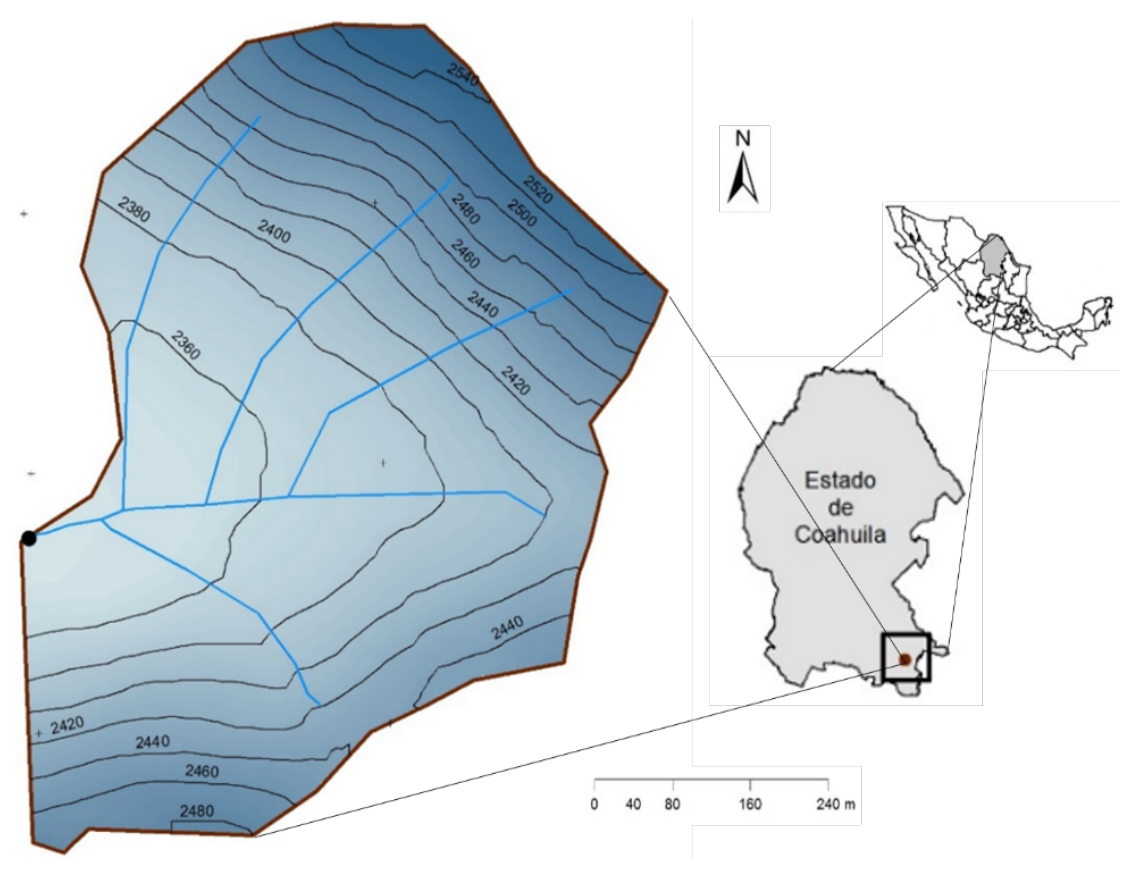

Fuente: Inegi, 2017.

Figura 1. Cuenca Experimental de Saltillo (CES) en Coahuila.

El clima, según la clasificación climática de Köppen, modificada por García (1988), corresponde al tipo $\mathrm{BS}_{1} \mathrm{kw}$, semiseco templado, con temperaturas entre 11.5 a 25.3 ${ }^{\circ} \mathrm{C}$, con una precipitación promedio anual de $356.7 \mathrm{~mm}$ (Díaz et al., 2007). La vegetación es un bosque de Pinus cembroides Zucc. (pino piñonero) asociado con Quercus spp (encinos) con pastizal nativo (Figura 2) integrado por las siguientes taxa: Larrea tridentata (DC.) Coville (gobernadora), Yucca carnerosana (Trel.) McKelvey (palma samandoca), Yucca filifera Chabaud (palma china), Fouquieria splendens Engelm. (ocotillo), Juniperus sp (táscate), Agave lechuguilla Torr. (lechuguilla), Opuntia spp (nopal), Dalea bicolor Humb. \& Bonpl. ex Willd. (engordacabra), Flourencia cernua DC. (hojasén), Atriplex canescens (Pursh) Nutt. (costilla de vaca), Agave spp (maguey), Acacia farnesiana (L.) Willd. (huizache), Euphorbia antisyphilitica Zucc. (candelilla), Mimosa biuncifera Benth. (gatuño), Dasylirion cedrosanum Trel. (sotol), Parthenium argentatum Gray (guayule), 
Bouteloua gracilis (Kunth) Lag. ex Steud. (zacate navajita), B. curtipendula (Michx)Torr (banderita), Lycurus phleoides Kunth (lobero) y Hilaria sp (grama).

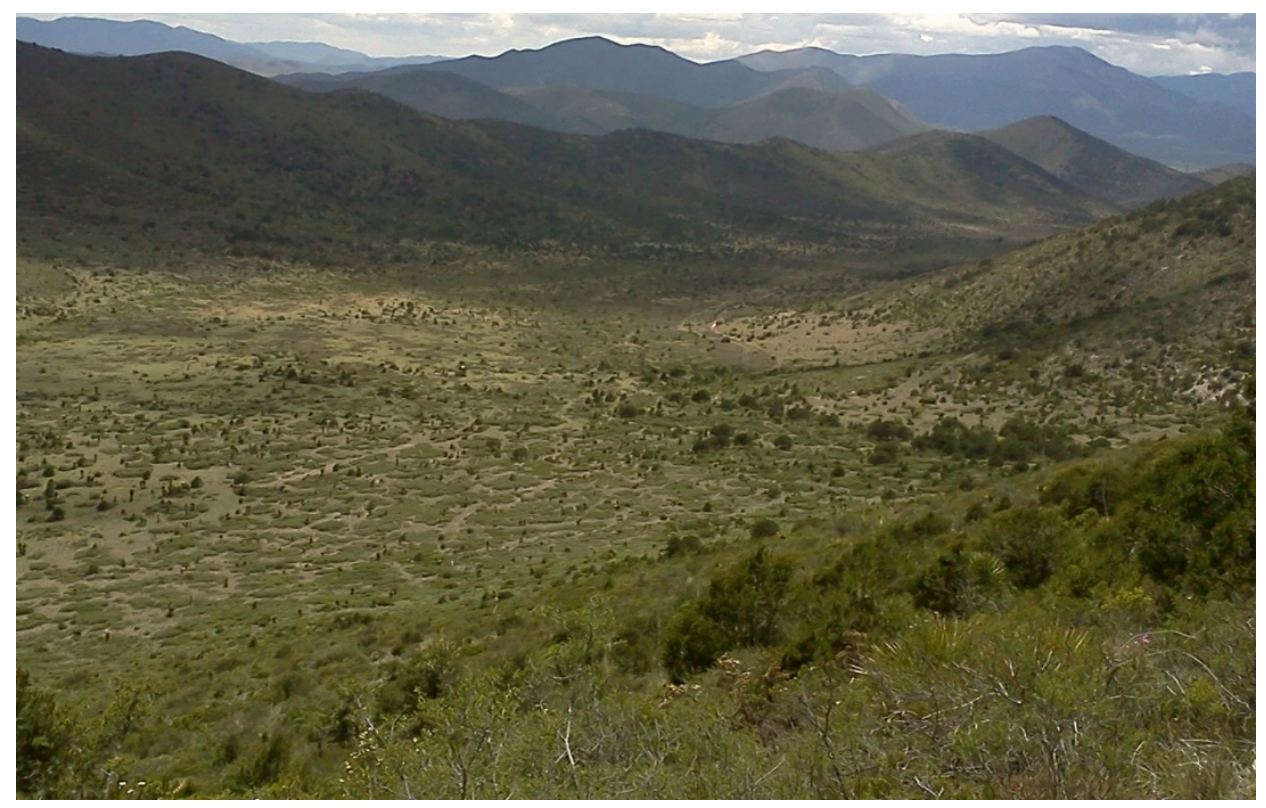

Figura 2. Panorámica general de los tipos vegetación en la Cuenca Experimental Saltillo, Coah.

La pendiente media del terreno en la cuenca es de $50.7 \%$, con una altitud promedio de 2450 m; las unidades de suelo (FAO-UNESCO, 1990) son Leptosol calcárico y Feozem esquelético de textura media con fragmentos de grava en la superficie y de fertilidad media. Los recursos naturales en la Cuenca enfrentan problemas como la escasa cobertura vegetal en laderas, la erosión de laderas, la invasión de especies indeseables para la ganadería y la formación de cárcavas.

Registro de variables hidrológicas. Con el propósito de cuantificar algunas de las variables hidrológicas, se pusieron diversos equipos para el monitoreo temporal de las mismas. Con respecto al clima, se instaló una estación meteorológica automatizada ADCON Telemetry. Para el monitoreo de los escurrimientos superficiales, se construyó junto a la estación climatológica un canal de aforo tipo $\mathrm{H}$, 
en el cual se colocó un sensor óptico (de manufactura local, sin patente) para estimar la altura del flujo superficial con respecto al piso del canal (Figura 3).
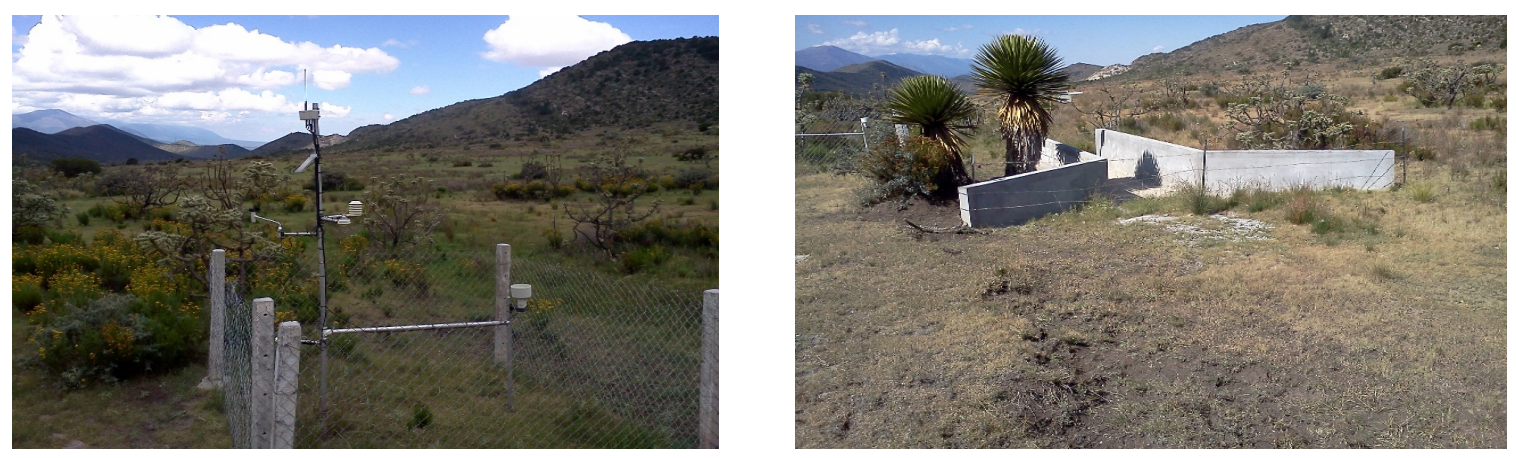

Figura 3. Estación climatológica y canal de aforo de escurrimientos superficiales en la Cuenca Experimental Saltillo, Coah.

\section{Régimen pluviométrico}

La caracterización termo-pluviométrica del clima, basada en los registros de la estación climatológica ubicada en la Cuenca Experimental, se presenta de manera gráfica en la Figura 4. El año 2016 se consideró "bueno", en términos de la cantidad de la lámina total anual precipitada $(606.6 \mathrm{~mm})$, con respecto al promedio anual (367.0 mm) registrado por Servicio Meteorológico Nacional. Para este año, se contabilizaron 151 eventos de precipitación (Iluvia máxima en 24 horas) mayores a un milímetro, de ellos, cinco registraron una intensidad superior a 10 milímetros, en 15 minutos. Se observan en el Cuadro 1 que a excepción de los meses de febrero, abril, julio, septiembre y octubre, la lámina mensual registrada fue mayor que la precipitación promedio mensual obtenido desde 1951 hasta 2010. En la época de Iluvia (de junio a septiembre), solo el mes de julio recibió $21 \%$ del promedio mensual histórico. 


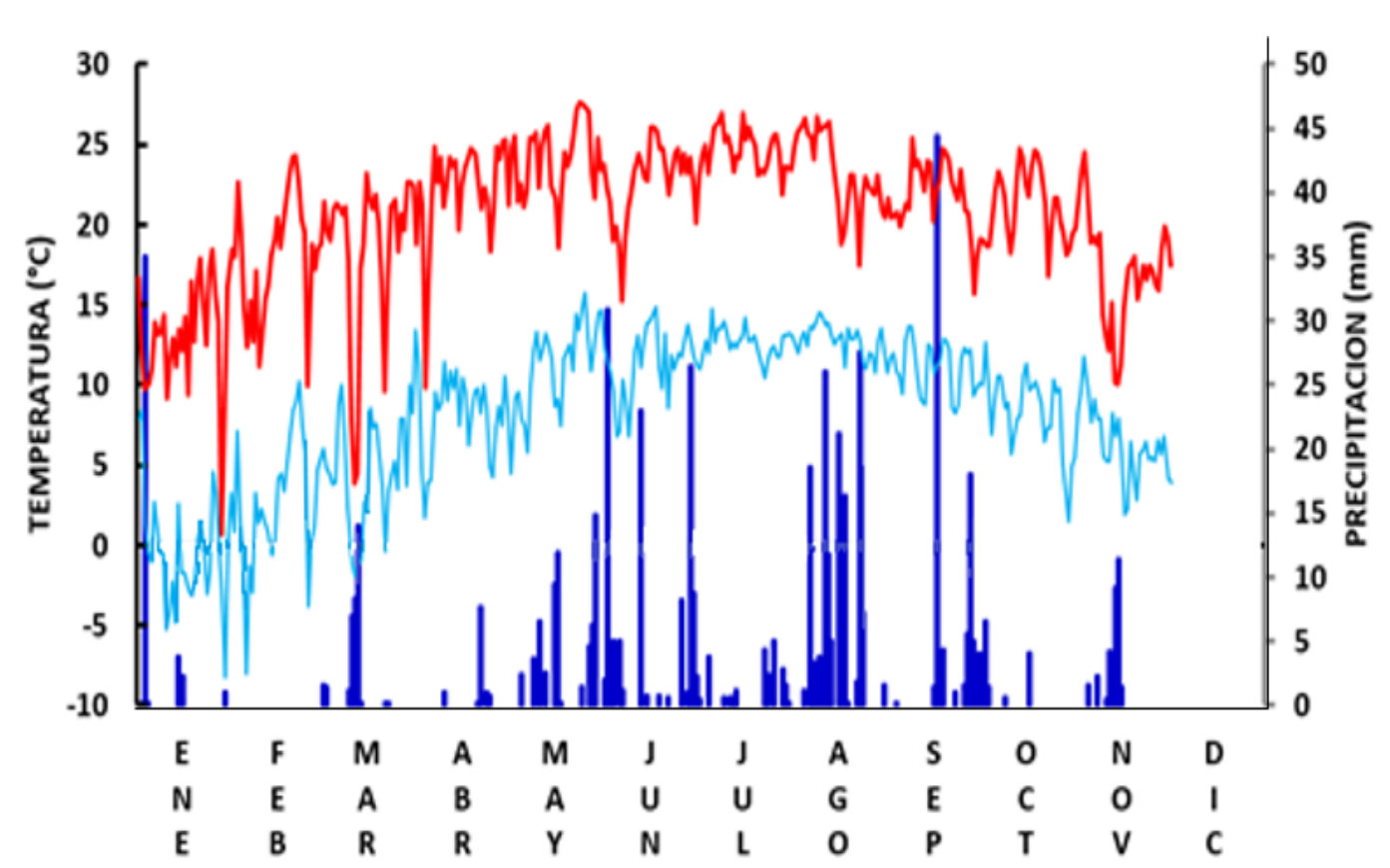

Temperatura máxima = Línea roja; Temperatura mínima = Línea azul claro;

Precipitación pluvial = Barra azul obscuro.

Figura 4. Régimen termo-pluviométrico en la Cuenca Experimental Saltillo, Coah., durante 2016.

Con los registros de La estación se obtuvieron algunos estadísticos de la serie de tiempo del año 2016, los cuales se presentan en el Cuadro 1. En cuanto a la persistencia de la ocurrencia o registro de la lluvia máxima en 24 horas (días continuos con registro de lluvia) se puede señalar que el mes con mayor persistencia de eventos de precipitación fue septiembre, con al menos 5 días continuos de lluvia.

Dado que los datos constituyen una serie histórica muy corta (un año) no se obtuvo una relación clara entre la lámina precipitada y la registrada los cinco y tres días previos ( $R^{2}=0.17$ y 0.39 , respectivamente); sin embargo, la línea de tendencia es negativa; lo que indica que, con eventos de mayor cantidad, la magnitud de los anteriores al mismo es muy baja. Es importante señalar que, para los dos sucesos 
registrados con más precipitación (44.4 y $30.8 \mathrm{~mm}$ ), la cantidad acumulada de Iluvia en los tres días previos fue de 1.4 y $2.0 \mathrm{~mm}$, respectivamente.

Ante la ocurrencia de lluvias de este tipo (chubascos) es común observar la respuesta hidrológica de las cuencas en forma de escurrimiento superficial. A pesar de lo anterior, al momento de presentarse esos eventos en particular, las condiciones de humedad edáfica, la capacidad de infiltración en sitios de cuenca con menor pendiente y mayor cobertura vegetal del suelo no fueron las óptimas para generar una lámina de flujo suficiente para ser registrada en la estación de aforo.

Con base en el Método de Curva Numérica, desarrollado por USDA-SCS (1972), respecto lo concerniente a la condición de humedad antecedente como función de la precipitación pluvial, se puede señalar que de los 80 eventos de lluvia superiores a un milímetro en 24 horas, $62.5 \%$ de las ocasiones en que se presentó uno, la condición de humedad antecedente en la cuenca fue I (la precipitación acumulada 5 días previos al evento de 0 a $12.7 \mathrm{~mm}$ ); $26.3 \%$, la condición fue II (la precipitación acumulada cinco días previos a la precipitación de 12.7 a $38.1 \mathrm{~mm}$ ); y solo $11.2 \%$ estuvo en condición III (la precipitación acumulada cinco días previos al evento > $38.1 \mathrm{~mm}$ ). En términos de humedad antecedente y de manera genérica, las condiciones del suelo en esta parte de la cuenca corresponden a la condición seca (CN I). 
Cuadro 1. Estadísticos de los eventos diarios de precipitación pluvial en la estación climatológica de la Cuenca Experimental Saltillo, Coah. (2016).

\section{Histórico}

1951-2010*

2016

\begin{tabular}{|c|c|c|c|c|c|}
\hline \multirow{2}{*}{ Mes } & \multicolumn{4}{|c|}{ Precipitación pluvial } & \multirow{2}{*}{$\begin{array}{c}\text { ETA } \\
\begin{array}{c}\text { Potencial } \\
\Omega\end{array} \\
\begin{array}{c}\text { Suma } \\
(\mathbf{m m})\end{array}\end{array}$} \\
\hline & $\begin{array}{c}\text { Promedio } \\
(\mathbf{m m})\end{array}$ & $\begin{array}{l}\text { Suma } \\
(\mathbf{m m})\end{array}$ & $\begin{array}{c}\text { Lluvia máxima } \\
\text { en } 24 \text { h } \\
(\mathrm{mm})\end{array}$ & $\begin{array}{l}\text { Persistencia } \\
\text { (días) }\end{array}$ & \\
\hline Enero & 15.1 & 42.4 & 35.0 & 2 & 80.2 \\
\hline Febrero & 12.2 & 0.0 & 0.0 & 0 & 94.9 \\
\hline Marzo & 8.3 & 34.2 & 14.0 & 4 & 103.2 \\
\hline Abril & 16.9 & 10.6 & 7.6 & 1 & 123.2 \\
\hline Mayo & 30.6 & 65.8 & 14.8 & 3 & 135.0 \\
\hline Junio & 46.4 & 117.6 & 30.8 & 4 & 133.3 \\
\hline Julio & 61.4 & 23.6 & 5.0 & 2 & 126.4 \\
\hline Agosto & 57.1 & 155.4 & 27.6 & 5 & 113.0 \\
\hline Septiembre & 64.1 & 86.0 & 44.4 & 4 & 106.2 \\
\hline Octubre & 30.1 & 14.8 & 6.6 & 3 & 107.1 \\
\hline Noviembre & 11.7 & 31.2 & 11.4 & 3 & 76.2 \\
\hline Diciembre & 13.1 & 25.0 & 0.0 & 3 & 71.8 \\
\hline Acumulado & 367.0 & 606.6 & & & 1270.6 \\
\hline
\end{tabular}

$*=\mathrm{SMN} ; \Omega=$ Evapotranspiración potencial registrada en la cuenca

\section{Hidrología superficial}

Generalmente, la respuesta hidrológica en cuencas forestales está en función, entre otras cosas de la magnitud y duración de las tormentas (Ide et al., 2010). Como se indicó, anteriormente, en la Cuenca Experimental durante 2016 no se tuvieron 
eventos de escurrimiento superficial directo en la estación de aforo. Se deduce que la totalidad del agua precipitada que escurrió de las laderas, se infiltró en la parte baja de la cuenca; debido a que la magnitud e intensidad de la precipitación no fue lo suficientemente alta para sobrepasar la tasa de infiltración del suelo en dicha zona (el valle) de la cuenca. Aunque, en cuencas forestales es común la presencia de flujo preferencial (Alaoui et al., 2011), en el valle de la cuenca en estudio dominan los pastizales y vegetación arbustiva, cuyas raíces no tienen un impacto importante en la formación de macroporos en la matriz del suelo.

Otro factor relacionado con la ausencia de flujo superficial es el contenido de humedad del suelo, previo al evento de precipitación (La Torre et al., 2011). La humedad edáfica para un tiempo determinado es la resultante del balance hídrico del sitio (Bay, 1969). En la Cuenca Experimental, debido a la baja persistencia de los eventos de precipitación y a la evapotranspiración observada en los meses de la época lluviosa (Cuadro 1) indican que las condiciones durante 2016 no fueron propicias para generar el escurrimiento superficial; la evidencia es el nulo registro de escurrimiento superficial.

Desde el punto de vista de la conservación del suelo y agua, este comportamiento es idóneo; es decir, en la cuenca existen las condiciones del suelo (textura arenosa en promedio para 25 sitios de muestreo, en promedio el contenido de arena fue superior a $33 \%$ ) y excelente cobertura basal ( $89 \%$ por gramíneas, herbáceas y mantillo orgánico) para infiltrar una lámina precipitada de $44 \mathrm{~mm}$, en dos horas con 15 minutos, con una intensidad máxima de $70 \mathrm{~mm}$ $\mathrm{hr}^{-1}$ (Figura 5). Comportamiento hidrológico QUE puede minimizar la energía erosiva proporcionada por el flujo superficial en la parte plana de la cuenca; Hernández et al. (2013) coinciden, al señalar la escasa presencia de cárcavas en sitios de pastizal cercanos a la cuenca experimental.

Los afectados directos de la respuesta hidrológica de la Cuenca Experimental son los ganaderos, ya que la falta de escurrimientos superficiales reduce la posibilidad de 
almacenar el agua en bordos, para el consumo de los animales. Esto conlleva a que los productores busquen cómo incrementar los coeficientes de escurrimiento, para aumentar la producción del preciado líquido, aunque sea en perjuicio de la conservación del suelo, e incluso de la vegetación presente en la cuenca.

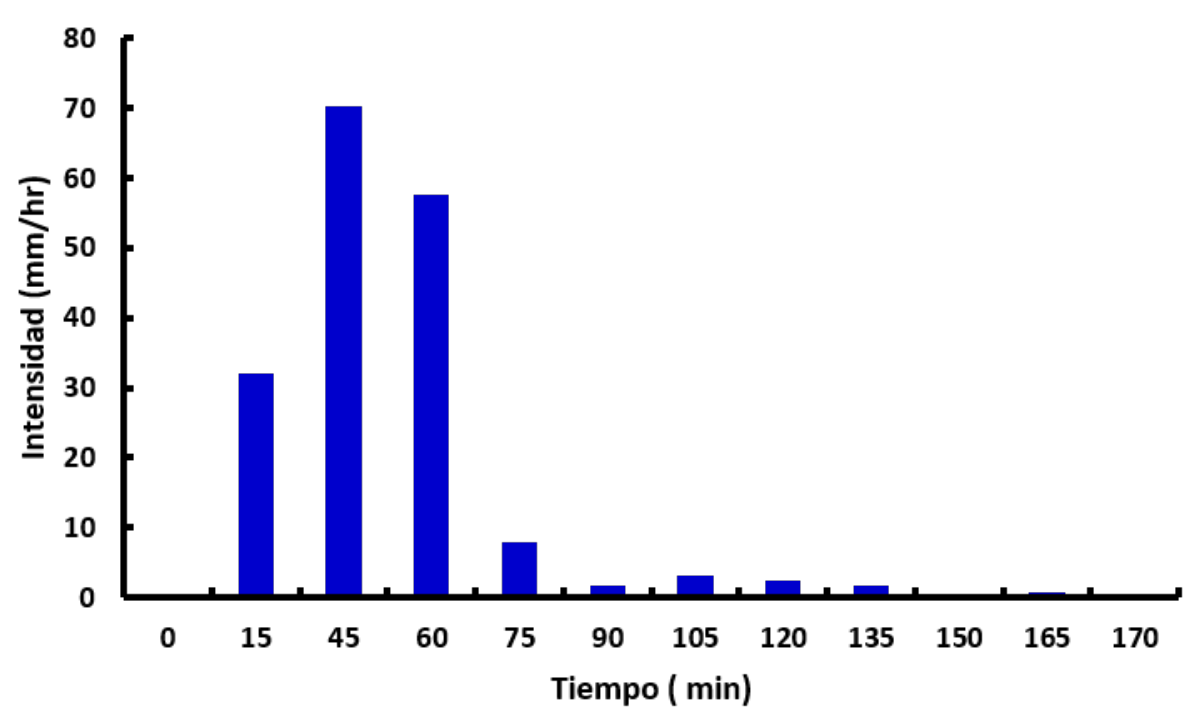

Figura 5. Distribución temporal de la intensidad del evento de lluvia ocurrido el 15 de septiembre de 2016 en la Cuenca Experimental Saltillo, Coah.

\section{Modelación hidrológica}

Con la finalidad de explicar la respuesta hidrológica de la cuenca experimental, se utilizó el modelo WIN TR - 55 para simular, de manera distribuida, la generación de escurrimiento superficial en la cuenca, de acuerdo a sus condiciones actuales. Esta se dividió en cuatro unidades con respuesta hidrológica (URH), como se muestra en la Figura 6. Las condiciones del terreno en cada unidad (Cuadro 2) pueden diferenciarse con base en la superficie, fisiografía (ladera o valle); la condición hidrológica, en función de la cobertura por los pastos y grupo hidrológico (capacidad 
del suelo y vegetación para generar escurrimiento superficial). A partir de esos aspectos, se asignó a cada área un valor de curva numérica, según el manual del USDA-SCS (1972). Un elemento para diferenciar cada URH fue su vegetación actual; por ejemplo, aunque la vegetación de toda la cuenca corresponde a bosque de coníferas asociado con matorral xerófilo, para el URH 1 en su mayor parte corresponde a matorral xerófilo, con suelo degradado y una pendiente promedio de $33.5 \%$; la URH 2 está representada por coníferas y gramíneas con presencia de cárcavas, una pendiente de $41.9 \%$; la URH 3, es la parte de la cuenca con más cobertura vegetal, principalmente, integrada por especies de Quercus, en un terreno con cobertura basal conformada por mantillo orgánico y fragmentos rocosos, una pendiente de $26 \%$; y la URH 4 corresponde al valle de la cuenca, en el cual el estrato basal está dominado por matorral xerófilo y pastos, con una pendiente de $8.9 \%$. Es importante señalar que a excepción de la URH 4, el resto de la superficie constituye un área de captación de agua de lluvia y fuente de escurrimientos superficiales; mientras que, para la producción de sedimentos, toda la superficie de la cuenca tiene la posibilidad de presentar pérdidas de suelo, en mayor grado la URH 2. 


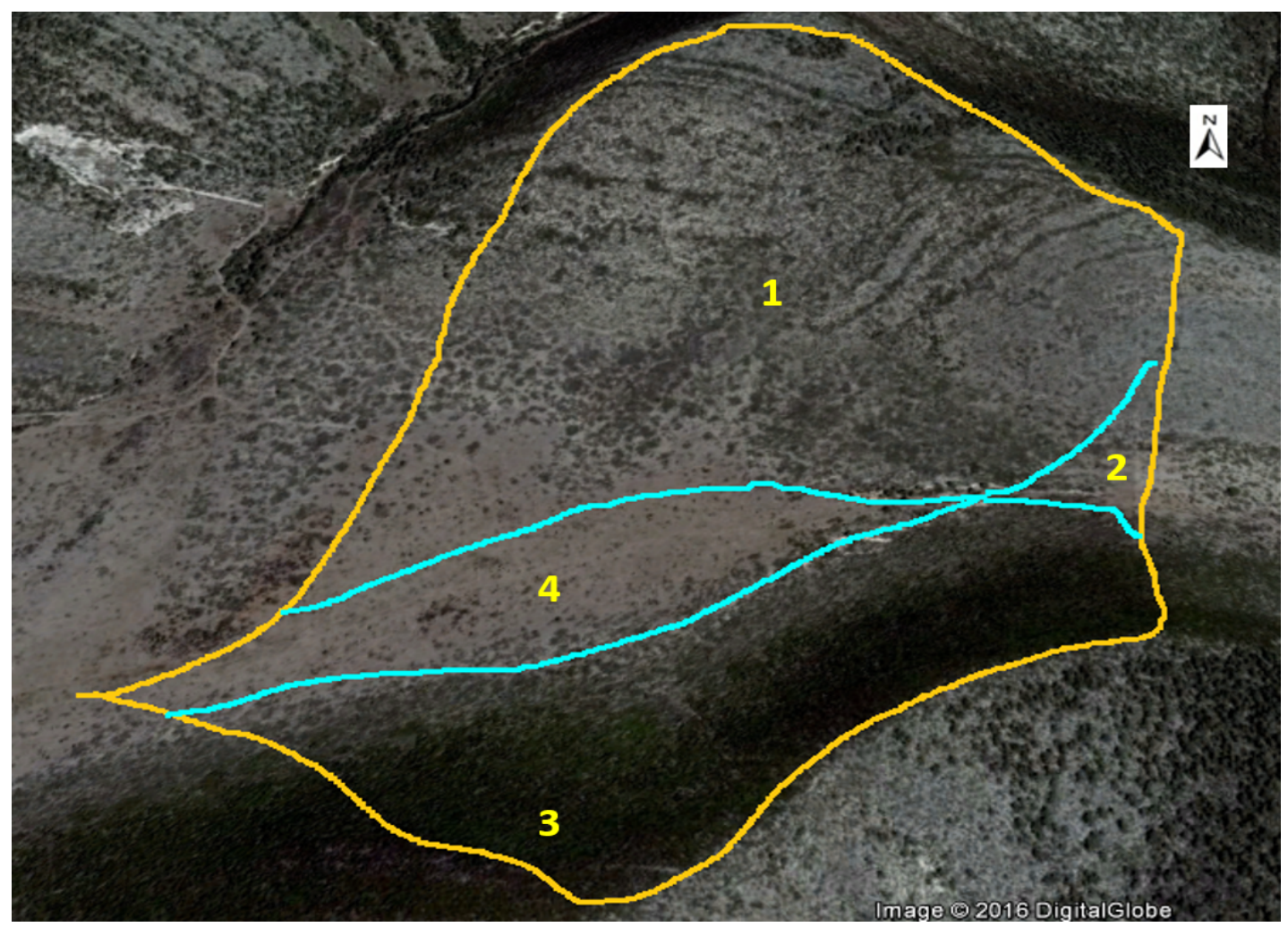

Figura 6. Representación esquemática de las Unidades de Respuesta Homogénea (URH) en la Cuenca Experimental Saltillo, Coah.

Cuadro 2. Caracterización y parámetros del Modelo WIN TR - 55 para cada una de las áreas de la Cuenca Experimental Saltillo, Coah. (2016).

\begin{tabular}{|c|c|c|c|c|c|c|}
\hline \multirow[b]{2}{*}{ Área } & \multirow{2}{*}{$\begin{array}{c}\text { Superficie } \\
\text { (ha) }\end{array}$} & \multirow[b]{2}{*}{ Fisiografía } & \multicolumn{2}{|c|}{ Condición hidrológica } & \multirow{2}{*}{$\begin{array}{c}\text { Grupo } \\
\text { hidrológico } \\
\text { de suelo }\end{array}$} & \multirow{2}{*}{$\begin{array}{c}\text { Curva numérica } \\
\text { ponderada }\end{array}$} \\
\hline & & & Condición & $\begin{array}{c}\text { Cobertura } \\
\qquad(\%)\end{array}$ & & \\
\hline URH1 & 22.9 & Ladera & Regular & 50 a 75 & B & 58 \\
\hline URH2 & 3.1 & Ladera & Regular & 50 a 75 & B & 58 \\
\hline URH3 & 14.9 & Ladera & Buena & $>$ de 75 & B & 55 \\
\hline URH4 & 6.2 & Valle & Buena & $>$ de 75 & B & 61 \\
\hline
\end{tabular}


Los resultados de las simulaciones del escurrimiento superficial de la Cuenca Experimental con el Modelo WIN TR - 55 coinciden con los registros obtenidos en la estación de aforo; es decir, que aun con la lluvia de mayor magnitud registrada en la estación climatológica, del sitio en $2016(44 \mathrm{~mm})$, el modelo no generó escurrimiento, algo similar se observó en campo. Para que se registre flujo superficial a la salida de la cuenca, se requiere que exista una lámina precipitada, en 24 horas, superior a $65 \mathrm{~mm}$ (lluvia con un periodo de retorno de 25 años) según lo publicado por la SCT (1990) (Figura 7).

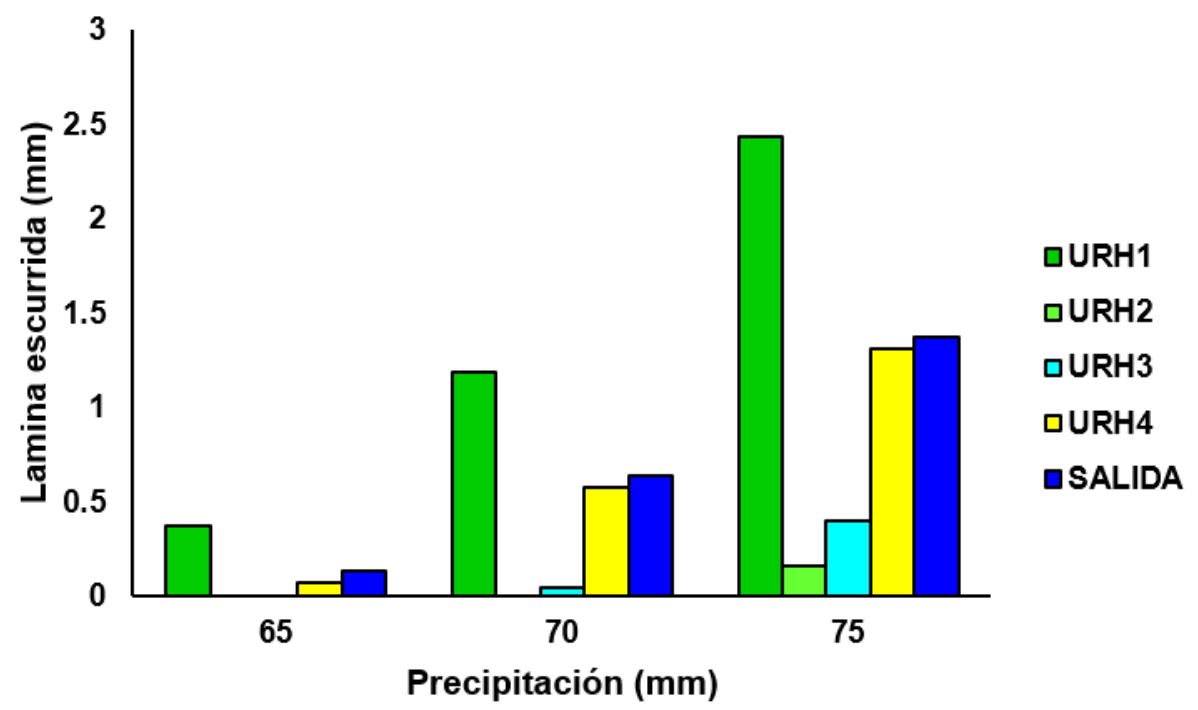

Figura 7. Relación entre la lámina escurrida $(\mathrm{mm})$ simulada por el Modelo WIN TR - 55 en las diferentes URH y a la salida de la cuenca, para diferentes magnitudes de eventos de precipitación en la Cuenca Experimental Saltillo, Coah.

La simulación del comportamiento hidrológico de la Cuenca Experimental evidencia la existencia de un efecto de área de aportación de escurrimiento (Figura 7). Para esta cuenca, el Área 1 es la parte que más contribuye a la ocurrencia del flujo superficial; por su mayor superficie, pendiente, escasa cobertura vegetal y 
profundidad del suelo. La segunda área fuente de escurrimiento es la parte de la cuenca considerada, fisiográficamente, como el valle; superficie que a pesar de tener menor pendiente que las otras tres, por su cercanía a la estación de aforo, porque recibe las aportaciones del exceso de lluvia de las otras partes, y cuando los contenidos de humedad en el suelo son altos, previos a la tormenta al evento torrencial: el valle se considera el área que más contribuye al escurrimiento que potencialmente se registraría en el canal de aforo, a la salida de la cuenca.

Los valores de las curvas numérica utilizados en este estudio coinciden con el intervalo registrado para otras cuencas forestales (Tedela et al., 2012); sin embargo, a pesar de que no fue el objetivo a evaluar el método del SCS es importante señalar que debido a que los valores utilizados son tabulares es necesario realizar investigación para definir con más precisión y mediante un análisis de sensibilidad, el valor de la curva numérica para cuencas forestales (Tedela et al., 2012), en las cuales existe un diferencial de tipos de suelo (por efecto de erosión hídrica), de gradiente altitudinal (pendiente del terreno), génesis de suelos (suelos aluviales), así como del tipo y estado de sucesión vegetal en cuencas afectadas por mal manejo o incendios.

\section{Conclusiones}

La información generada es preliminar, por lo que se requiere incrementar la base de datos de clima, suelo y vegetación. Lo anterior obliga a dar continuidad al seguimiento de variables hidrológicas en la Cuenca Experimental Saltillo, Coah.

La nula respuesta hidrológica de la cuenca, en términos de flujo superficial, durante 2016 es un indicador de la interacción del manejo de los recursos agua-sueloplanta-animal con las características climatológicas y las condiciones físicas de la cuenca. En este contexto, es importante utilizar técnicas como los trazadores 
químicos para determinar los movimientos de los flujos subterráneos del agua y su posible contribución al incremento de fuentes de agua para la ciudad de Saltillo.

Analizar como la variabilidad espacial y temporal de la intensidad de la lluvia, el proceso de infiltración, la redistribución de la humedad en el suelo por áreas de escurrimiento; así como, la dinámica de la cobertura vegetal del suelo en función de su manejo. Por otro lado, es importante precisar los valores de la curva numérica y validar la información hidrológica generada por el Modelo WIN TR - 55 con respecto de la generación del escurrimiento a partir de eventos de precipitación mayores a $65 \mathrm{~mm}$ en cuencas forestales.

\section{Agradecimientos}

El presente trabajo forma parte del Proyecto Fiscal del INIFAP: "Monitoreo de procesos físicos en cuencas hidrológicas de zonas marginadas y con degradación ambiental" (Núm. de Proyecto SIGI: 1050332913). Se agradecen las aportaciones de los revisores técnicos; cuyas observaciones contribuyeron a la mejora de la calidad de esta nota de investigación.

\section{Conflicto de intereses}

Los autores declaramos no tener conflicto de intereses 


\section{Contribución por autor}

Miguel A. Velásquez Valle: análisis hidrológico; Gerardo Esquivel Arriaga: determinación de parámetros geomorfológicos de la cuenca; Ignacio Sánchez Cohen: análisis hidrológico; David Castillo Quiroz: identificación de la vegetación; Antonio Cano Pineda: análisis de información climatológicos; Ramón Gutiérrez Luna: muestreo e identificación de la vegetación.

\section{Referencias}

Alaoui, A., U. Caduff, H. H. Gerke and R. Weingartner. 2010. Preferential Flow Effects on Infiltration and Runoff in Grassland and Forest Soils. Vadose Zone Journal $10: 367-377$.

Bay, R. R. 1969. Runoff from small peatland watersheds. Journal of Hydrology 9: 90-102.

Černohous, V. and P. Kovář. 2009. Forest watershed runoff changes determined using the unit hydrograph method. Journal of Forest Science 55(2): 89 - 95.

Chen, L., M. Berli and K. Chief. 2013. Examining Modeling Approaches for the Rainfall-Runoff Process in Wildfire-Affected Watersheds: Using San Dimas Experimental Forest. Journal of the American Water Resources Association 49(4): 851-866.

Cui, X., S. Liu and X. Wei. 2012. Impacts of forest changes on hydrology: a case study of large watersheds in the upper reaches of Minjiang River watershed in China. Hydrology and Earth System Science 16: 4279-4290.

DeBano, L. F. 2009. Fire effects on watersheds: An overview. Southwest Hydrology. University of Arizona. Tucson, AZ, USA. pp. 28-29. 
Díaz P., G., J. A. Ruiz C., G. Medina G., M. A. Cano G., V. Serrano A. e I. Sánchez C. 2007. Estadísticas climatológicas básicas del estado de Coahuila (Período 19612003). INIFAP-CIRGOC. Xalapa, Ver., México. Libro Técnico Núm. 161. 159 p.

Diario Oficial de la Federación (DOF). 2014. Programa Nacional Forestal 2014-2018. Segob. 28 de abril de 2014.

http://dof.gob.mx/nota_detalle.php?codigo=5342498\&fecha $=28 / 04 / 2014$

(2 de marzo de 2017).

Organización de las Naciones Unidas para la Alimentación-Organización de las Naciones Unidas para la Educación, Ciencia y Cultura (FAO-UNESCO). 1990. Mapa mundial de suelos, leyenda revisada. Versión en español, preparada por la Sociedad Española de la Ciencia del Suelo. Roma, Italia. 152 p.

García, E. 1988. Modificaciones al sistema de clasificación climática de Köppen. Instituto de Geografía. Universidad Nacional Autónoma de México. México, D.F., México p. 246

Gholami, V. 2013. The influence of deforestation on runoff generation and soil erosion (Case study: Kasilian watershed. Journal of Forest Science 59 (7): $272-278$.

Gobierno del Estado de Coahuila. 1998. Programa de manejo de la zona sujeta a conservación ecológica "Sierra de Zapalinamé". Secretaría de Desarrollo Social. Dirección General de Ecología. Saltillo, Coah., México. 282 p.

Gobierno de Coahuila. 2012. Agua potable, drenaje y saneamiento 2011 - 2017. Programa Coahuila de Zaragoza. http://coahuila.gob.mx/archivos/pdf/Publicaciones/PROGRAMA\%20ESPECIAL\%20D E\%20AGUA\%2011-17.pdf (16 de febrero de 2017).

Hernández A., R., R. Vázquez A., J. Valdes R. y R. López T. 2013. Comparación de los métodos de condición y salud del pastizal. Agraria 10 (1): 27 - 36. 
Hlásny, T., D. Kočický, M. Maretta, Z. Sitková, I. Barka, M. Konôpka and H. Hlavatá. 2015. Effect of deforestation on watershed water balance: hydrological modellingbased approach. Lesnicky časopis Forestry Journal 61: $89-100$.

Ide, Y. Shinohara, N. Higashi, H. Komatsu, K. Kuramoto and K. Otsuki.2010. A preliminary investigation of surface runoff and soil properties in a moso-bamboo (Phyllostachys pubescens) forest in western Japan. Hydrological Research Letters. 4: 80-84.

La Torre T., I. B., D. M. Amatya, G. Sun and T. J. Callahan. 2011. Seasonal rainfallrunoff relationships in a lowland forested watershed in the southeastern USA. Hydrological Processes 25:2032-2045.

Rodríguez-Trejo, D. A. y Z. P. Fulé. 2003. Fire ecology of Mexican pines and a fire management proposal. International Journal of Wildland Fire 12: $23-37$.

Sánchez S., J. A., L. M. Torres E., A. Cano P. y O. U. Martínez B. 2003. Daños y diversidad de insectos descortezadores de coníferas del noreste de México. Revista Ciencia Forestal en México 28 (93): 41 - 56.

Sánchez C., I. 2005. Fundamentos para el manejo integral del agua. Instituto Nacional de Investigaciones Forestales, Agrícolas y Pecuarias. CENID-RASPA. Gómez Palacio, Dgo. México. Libro Científico Núm. 2. 272 p.

Secretaría de Comunicaciones y Transportes (SCT). 1990. Isoyetas de intensidadduración frecuencia. República Mexicana. Subsecretaría de infraestructura. México, D. F., México. 495 p.

Secretaria de Medio Ambiente y Recursos Naturales - Comisión Nacional Forestal (Semarnat-Conafor). 2013. Inventario Estatal Forestal y de Suelos. Coahuila 2013. Semarnat. México, D.F., México. 137 p.

Swindel, B. F., C. J. Lassiter and H. Reikerk. 1983. Effects of clearcutting and site preparation on stormflow volumes of streams in Pinus elliottii flatwoods forests. Forest Ecology and Management 5(4): 245 - 253. 
Tedela, N. H., S. C. McCutcheon, T. C. Rasmussen, R. H. Hawkins, W. T. Swank, J. L. Campbell, M. B. Adams, C. R. Jackson and E. W. Tollner. 2012. Runoff Curve Numbers for 10 Small Forested Watersheds in the Mountains of the Eastern United States. Journal of Hydrologic Engineering 17(11):1188-1198.

Torres-Rojo, J. M. and R. Flores-Xolocotzi. 2014. Deforestation and land use change in Mexico. Journal of Sustainable Forestry 12(1-2):171 - 192.

U.S. Department of Agriculture-Soil Conservation Service (USDA-SCS). 1972. SCS. Hydrology. In: Mockus, V. (ed.). Chapter 10. Estimation of direct runoff from storm rainfall. U.S. Department of Agriculture, Soil Conservation Service. Washington, DC USA. National Engineering Handbook. Section 4. 25 Report. pp. 10.1-10.24.

U.S. Department of Agriculture - Natural Resources Conservation Service (USDANRCS). 1986. Urban hydrology for small watersheds. U.S. Department of Agriculture, Conservation Engineering Division. Washington, DC USA. Technical Release 55. 164 p.

Yu, P., Y. Wang, N. Coles, W. Xiong and L. Xu. 2015. Simulation of Runoff Changes Caused by Cropland to Forest Conversion in the Upper Yangtze River Region, SW China. PLOS ONE 10(7): e0132395. doi: 10.1371/journal.pone.0132395.

Zheng, H., F. Chen, Z. Ouyang, N. Tu, W. Xu, X. Wang, H. Miao, X. Li and Y. Tian. 2008. Impacts of reforestation approaches on runoff control in the hilly red soil region of Southern China. Journal of Hydrology 356: 174-184. 